\title{
Discriminately disposable after single use: the trophectoderm and its derivatives
}

\author{
David F. Albertini ${ }^{1}$
}

Published online: 5 January 2016

(C) Springer Science+Business Media New York 2016

With the start of a new year, reflective cogitation prompts the belief that change from the immediate past will bear fruits of future gains. So it is that we launch 2016 here at JARG with an attitude embracing the need for better discrimination between new knowledge of a translational import and the adoption of nascent technologies in the fields of human ARTs and genetics of human reproduction.

Leading off this issue, readers will be greeted by the opinion piece of Luke and colleagues posing a familiar and contentious question as growth in the ART marketplace continues and takes the form of a mosaic of approaches globally (Is the wrong question being asked in infertility research? 10.1007/ s10815-015-0610-3).

And for those looking to sustain the head scratching, we feature two provocative contributions raising concerns once again over monozygotic twinning (MZT), consider from Vaughan and colleagues, the perplexing observation that MZTs take on an unexpected tendency to present at specific times of the year (Clustering of monozygotic twinning in IVF, this issue, 10.1007/s10815-015). And from our colleagues in China, the intriguing case report from studies of SETs showing just how plastic human embryos can be at the moment of lineage determination and sourcing of the various extraembryonic products of the conceptus taking

Capsule We launch the 2016 edition of JARG with a series of papers forecasting the application of new wave genetics down to the curious and perplexing concerns being raised over the incidence and origins of monozygotic twins and associated defects in lineage segregation in the human embryo.

David F. Albertini

DALBERTINI@kumc.edu

1 University of Kansas Medical Center, Kansas, KS, USA charge during ART pregnancies (Monochorionic quadramniotic and triamniotic pregnancies following single embryo transfers: two case reports and a review of the literature 10.1007/s10815-015-0611-2). While deviations from the norm are abundant during the saga that is early human development, definition of their developmental origins remains an enigma for many such cases and gives us pause for the complexity that lies within and exactly how our interventions influence outcomes as discussed by Korosec and collaborators (Independent factors influencing large-forgestation birth weight in singletons born after in vitro fertilization, this issue, 10.1007/s10815-015-0601-4).

That deviations in embryogenesis are rooted in the history of either the male or female gamete (or both) is fast becoming a tangible focus for future research. And within our pages this month, aficionados of the ovary will find that the most plastic of these-granulosa cells - takes center stage in a series of original research contributions on the multipotential character that serves as the lifeline for the oocyte before it is ovulated. Among these, we especially draw your attention to the paper from Bentov and collaborators in Toronto who take on the curious case of how follicular fluid manages to store such enormous quantities of estrogen and what they reveal as a mechanism that defies most traditional biochemical explanations for this problem (What maintains the high intrafollicular estradiol concentration in pre-ovulatory follicles? 10.1007/s10815-015). And finally, we note the inroads made by molecular genetics and diagnostics whose momentum is likely to persist throughout the coming year.

In keeping with the theme of the trophectoderm and its inevitable demise, discourse continues over the fortunetelling features of trophoblast biopsies and genetic testing. Prognostic value and interpretative challenges are discussed in the paper by Ebner and colleagues (Quantitative and qualitative trophectoderm grading allows for prediction of live 
birth and gender 10.1007/s10815-015-0609-9). And yet another report on the validity of PGS finds concordance between results obtained through the magical mystery tour known as Day 3-Day 5 (this issue, Confirmation rates of array-CGH in day-3 embryo and blastocyst biopsies for preimplantation genetic screening 10.1007/s10815-015).

Finally, and extending the reach of molecular genetics deep into the realm of reproductive tract diagnostics, Scott and colleagues open yet another chapter in efforts to ascertain the potential predictive utility (or futility in this case) of thrombophilic SNPs as valid biomarkers of disease potential
(Multiple thrombophilic single nucleotide polymorphisms lack a significant effect on outcomes in fresh IVF cycles: an analysis of 1717 patients 10.1007/s10815).

Join us in 2016 for what has all the makings of a rollercoaster ride to and through the journey from gametes to children. And with that, we welcome our newest board members who bring an ever deepening curiosity of the field of reproductive medicine and biology. We thank Bill Kearns, Rebecca Krisher, Irma Virant Klun, and Kara Goldman for joining the JARG team and bringing with them their specialized areas of expertise. 